\title{
Cuerpo Extraño Vegetal de la vía aérea
}

Drs. Pablo Valenzuela Fuenzalida. ${ }^{1}$ Sonia Samith. ${ }^{2}$ Hernán Palazuelos B.. ${ }^{3}$ Gustavo Aldunate N., Marcela Guerra B.. 5

\begin{abstract}
Four children with chest and pleuropuimonary patholugy associated with aspiration of grass mads were admited in Whe Robertis del Rio Hospital.

The clinical diagnosis, radiolugycal findings and management were analized.

The moat important fact. in our experience, was a poud anamnesis. When it is imposible to recover the foreing body hy inhalation. pustural drainage and broncuscopy, mofter treatment is necesary in the typicsl perforgtjue case.

In the management of the dermal exirusion. conservatism is of ulmost importance.
\end{abstract}

La aspiración de cuerpos extraños dentro de la vía aérea constituye una emergencia médico quirúrgica frecuente, especialmente en loś primeros años de la

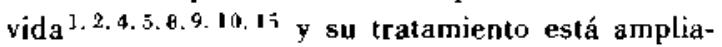
mente revisado. $5.2 .11,13$

La patología de los procesos que se suceden a la aspiración fue establecida por Chevalier Jackson en 1926." con especial referencia a los vegetales, los cuales por su movilidad y caracteristicas fisico quimicas producen los mayores trastornos.

La historia de la Medicina está jalonada de episodios y procedimientos ingeniosos para salvar al paciente que se encuentra en tan grave trance.

Aun cuando fue Hipucrates el primero en sugerir la intubación laríngea y traqueal, no fue sino hasta 1801 cuando $M$. Desault demostró que podría mantenerse un tubo de alimentación dentro de la traquea por tiempo prolongado.

I Unidar de Emergencias Respiratorias y Laboratorio Cardiorrespi. ratorio. Hospital Roberto del Rúo.

${ }^{2}$ Jefe Servicio de Radiología. Huspital Roberto del Rio.

3Otorrinolaringolozía, Hospital Roberto del Hio.

${ }^{4}$ Cirugia. Hospital Roberto del Rio.

"Becada de Radiología. Huspiral Roberto del Rio.

${ }^{6}$ Kinesiterapia. Hospital Roberto del Río.
En 1854 Manuel García introdujo el Laringoscopio de Espejo. En 1895 Kirstaein efectuó la primera laringoscopia directa y ese mismo año. Killian logró extraer, por primera vez, un cuerpo extraño desde un bronquio de un hombre de 63 años. Posteriormente, al inventarse la iluminación distal de los instrumentus. los procedimientos endoscópicos se hicieron habituales y el tratamiento de asfixia por aspiración redujo su mortalidad en forma espectacular. ${ }^{13}$

El propósito de la presente comunicación es el analizar la aspiración de cuerpos extraños vegetales y en especial la de inflorescencia (espigas), las que pueden ocasionar cuadros clínicos de larga evolución y particulares características clínicas y terapéuticas.

\section{CASOS CLINICOS}

Cașo N. 1.

L.B.E. 405720; Masculino; $6 / 4$ años.

24/XI/78: 8 días antes de su ingreso, jugando, aspira una espiga. Presenta una crisis de asfixia, con inten- 
sa difieultad respiratoria. dolor retroestemal y tos seca permanente. Es llevado al médico, que solicita un estudio radiolígico de torax. el cual es informado como "sombra tenue, difusa, en lóbuto inferior derecho con cisuritis y pleuritis marginal derecha". Se plantea la necesidad de efectuar una broncoscopia la cual debe diferirse por razanes extra médicas. 7 dias después se presenta una fluxión de la región costal derecha, con dolor local y evolución afebril. Se decidió esperar el drenaje espontáneo. Al $9^{\prime \prime}$ día de la hospitalización se inicia la eliminación espontánea de la espiga. la cual se completa al cabo de seis días. siendo dado de alta en buenas condiciones generales. (dia 23)

A los 30 dias de la aspiración, el control radiológico muestra imágenes insterticiales tenues en la base derecha con leve engrosamiento pleural marginal del mismo lado.

\section{Caso N. 2.}

M.G.O. 519810, masculino. 8 años.

10/11/78: Un mes ante de su ingreso aspira una espiga de pasto, presentando un episodio asfíctico seguido de tos productiva, síndrome febril y compromiso general. Fue tratado con Penicilina Sódica. Pur persistir la sintomatología de adinamia, astenia, anurexia y gran malestar general. es visto nuevamente por el médico quien diagnostica "pleuroneumonia derecha, draumatismo abdominal? siendo referido a los cirujanos. Estos plantean la posibilidad de un hematoma organizado, pues a nivel de la región costal baja aparece un aumento de volumen de curso progresivo. Referido al radiólogo, éste, luego de una anamnesis adecuada hace el diagnóstico de absceso de necesidad por cuerpo extraño vegetal. Con este diagnóstico se hospitaliza para su tratamiento definitivo. Dos días después de su ingrego y un mes luego de la aspiración se drena quirúrgicamente el absceso el cual se da salida a $200 \mathrm{cc}$ de contenido pútrido, en cuyo interior se encontró una espiga de pasto. La herida drenó durante seis días, al cabo de los cuales cerró completamente. Vo se ha podido controlar posteriormente al niño, por lo que el caso no se da como cerrado.

Caso N." 3.

\section{M.B.A. 504936, Femenino, 1/3 añıs.}

7/12/74: 15 días antes de su ingreso se produce la aspiración de una espiga de pasto, produciéndose un intenso síndrome asfictico, durante el cual la madre logra extraer parte de la espiga desde la faringe de la niña. Vista posteriormente en forma ambulatoria. es tratada como una bronquitis aguda. A los quince días de la aspiración. hay intensa tos irritativa, progresivo malestar general y baja de peso. siendo caralogado como una bronconeumonía y referido al Servicio de Urgencia para hospitalización. El estudio radinlógico de ingreso muestra un pioneumotorax enquistado del lado derecho. Por este motivo se efectúa una torascostomía que da salida a líquido pútrido que al cultivo mostró la presencia de un Corynebacterium Serosis y un Estafilococo dorato patógeno. La presencia de una fístula pleurocutánea persistente obligó a la debridación quirúrgica con lo cual sólo se consiguió una mejoria parcial. A los 20 dias de efectuada la toracostumía aún persistía la supuración y a los 35 días de la misma 667 días de la aspiración del C.E.) se produce la salida egpontánea, a través de la fístula, de una parte de la espiga de pasto. Controles efectuados dos meses después muestran una atelectasia del lóbulo medio derecho. La broncografía permite apreciar una disminución de volumen y retracción del lóbulo medio e inferior derecho. Posteriormente, en varias oportunidades. se producen hemoptisis, una de las cuales la lieva a la anemia aguda decidiéndose el tratamiento quirurgico, el cual se efectúa a los seis meses de la aspiración de la espiga. El estudio anatomopatológico del lóbulo medio derecho se informó como "Neumonitis crónica por cuerpo extraño. Se encontró una parte de una espiga de pasto de $10 \times 5 \mathrm{~mm}$.

Dos años después de la aspiración presenta una bronconeumenía bilateral que es tratada en forma habitual con evolución hacia la mejoría total. Cuatro años después del accidente no hay sintomatología respiratoria ni signología radiológica de ningún tipo.

\section{Caso N. ${ }^{\circ} 4$}

\section{J.J.D. 544372, masculino, 12 años.}

4/4/78: Es hospitalizado para estudio por presentar espectoración hemoptoica y una radiografía de torax que mostraba una infiltración para cardiaca derecha con una zona central de aspecto nodular. Entre sus antecedenes destacaban: a lo 5 años fue hospitalizado por una bronconeumonia. A los 6 años presenta una hemoptisis que fue tratada sintomáticamente. A los 7 años presenta una hemoptisis masiva que lo lleva a la anemia aquda. En esa oportunidad presentaba además tos permanente. Se efectuó un estudio para TBC que resultó negativo. Entre los 7 y 12 años 
está asintomático. La reactivación de la sintomatologia de homoptisis y tos es la causa de la hospitalización. El primer díagnóstico planteado fue el de bronquiectasia, efectuándose una broncografía que muestra una dificultad en el llene del bronquio basal posterior derecho, sin bronquiectasias. Con el fin de descrtar un secuestro pulmonar, se solicitó una arteriografía aúrtica y pulmonar que resultan normales. Por falta de mayores antecedentes y por la antiguiedad del proceso. se plantea el diagnisticos de un Quiste Broncógeno y se decide operar, practicándose una resección del segmento basal pusterior derecho. El cultivo del absceso crónico que se encontró fue negativo. Anatomía Patológica inforinó "Neumonía crónica por cuerpo extraño vegetal (espícula de pastot. El paciente mostró una buena evolución post operatoria y los controles posteriores han sido normales.

\section{DISCUSION}

Aun cuando la aspiración de cuerpos extraños vegetales y en especial de inflorescencias es un hecho relativamente raro, su análisis es necesario debido a los problemas diagnósticos y terapéuticos que plantean. Las aspiraciones son más frecuentes en los niños pequeños ${ }^{l}$ preferentemente lactantes mayores y preescolares. Dado que las circunstancias en que estos accidentes se producen son más o menos características, es posible recomendar algunas medidas preventivas. Es de todos conocida la afición de los pequeños por introducirse objetos por los orificios naturales de las vías aéreas. Existe mayor riesgo durante el juego. el ejercicio, la risa o la simple alimentación. Hay que considerar también que las inflorescencias se producen en los meses de primavera y verano y que duran hasta el otoño. ${ }^{6}$ La prevención consiste fundamentalmente en alejar al grupo vulnerable de la situación de riesgo.

Gráfico 1.

\section{CUADRO CLINICO}

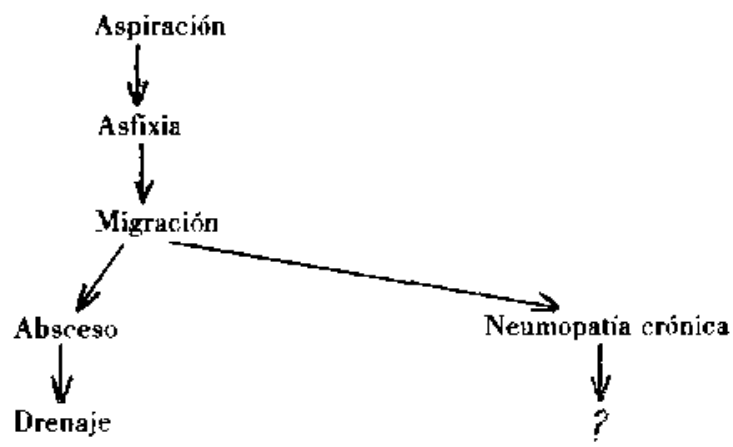

El gráfico $\mathrm{N} .{ }^{\circ}$ I nos muestra un resumen del cuadro clínico que presenta la aspiración de espigas de pasto del tipo penetrante: una vez que sucede la aspiración. se desencadena un cuadro de asfixia. Le siguen varios días asintomáticos. Posteriormente, periodo catalogado como de migración de la espiga, se suceden los diagnósticos de bronquitis, Bronconeumonía. Pleuroneumonía. Este periodo puede seguir dos caminos:

- ir al absceso y drenaje (evolución natural) Casos 1 y 2 .

- ir a la neumopatia crónica, en que se desconoce la evolución. Casos 3 y 4.

\section{Sospecha diagnóstica.}

Producida la aspiración, su diagnóstico puede ser dificil si no existe el antecedente anamnéstico claro, debiéndose sospechar la aspiración frente a una neumopatía de evolución arrastrada o recurtente, especialmente cuando se presenta el mismo segmento pulmonar. Debe sospecharse asimismo en la atelectasia crónica, bronquitis crónica, bronquectasia, absceso pulmonar y hemoptisis. 3.6, 12, 14, 16, 17

La radiología proporciona elementos de dianóstico muy importante en esta patología. Dado que el cuerpo extraño es radiolócido, el diagnóstico se basa en signos indirectos de obstrucción bronquial y en los provocados por la infección secundaria.

Debe recordarse que en todos los casos de enfermedad pulmonar infantil en los cuales no hay adecuada expansión pulmonar, la posibilidad de un cuerpo extraño debe tenerse en cuenta. Por otra parte. un examen radiológico normal no excluye el diagnóstico. Ante una historia concordante, debe seguirse el estudio con un examen broncoscópico.

En los casos de aspiración de espiga de pasto se han observado procesos de neumonitis de localización cambiante que corresponde al trayecto que sigue la espiga dentro del pulmón en su camino hacia la periferia. Puede así llegar hasta la pleura, originando un empiema que posteriormente se transformará en un absceso de necesidad de la pared torácica. Al romperse éste espontáneamente o por procedimientos quirúrgicos, se producirá la eliminación del C.E.

De cualquier modo, producida la sospecha de C.E. vegetal en la vía aérea, debe precisarse la naturaleza del mismo, pues cuando no se trata de una espiga netamente, ]a broncoscopía puede ser útil para su remoción. ${ }^{11,13}$

Si la broncoscopía no tuviese éxito y si no existiese la tendencia a la penetración. los procedimien- 
tos kinésicos son útiles, refiriendo algunos autores $^{2} \cdot 11,{ }^{13}$ un éxito en la eliminación de hasta un $25 \%$. Debe, sin embargo, insistirse en el riesgo que implica la migración o movilización de un C.E. en el árbol bronquial, pues, saliendo de un bronquio puede obstruir el otro, quedando de este modo el paciente sin posibilidad ventilatoria. Esta eventualidad obliga a realizar estos procedimientos en unidades que dispongan de elementos adecuados para la reanimación. tratamiento endoscópico y/o quirúrgico de urgencia.

La espiga aspirada tiende, como ya dijimos, a penetrar profundamente en el árbol bronquial, ayudada por los movimientos respiratorios y por su particular morfología, que le impide retroceder. Dicha migración, como ya se señaló llevará a la eliminación dentro de las próximas seis semanas, por medio de un absceso de necesidad. Este proceso se ubicará frecuentemente en los espacios inter-costales inferiores y contendrá pus de carácter pútrido con una flora bacteriana infrecuente en la vía aérea (Caso N. ${ }^{3}$ ).

Iniciado el proceso de expulsión, la eliminación espontánea es frecuentemente más efectiva que la intervención quirúrgica por lo que es aconsejable mantenerse a la expectativa. El empiema tabicado y/o la pleuritts localizada bacen más difícil la exploración quirúrgica y al interrumpir el proceso de eliminación, sólo se consigue, con frecuencia. prolongar el mismo, tal como sucedió en el caso N. ${ }^{9} 3$. Es preferible, por lo tanto, esperar que se establezca bien el absceso de necesidad y sólo drenar cuando haya gran fluctuación en piel. De ese modo se obtendrá el C.E. íntegro.

Los cuatro casos que presentamos permiten analizar la mayoría de las eventualidades que suceden luego de la aspiración de una influrescencia.

En el Caso N. ${ }^{\circ}$ l se aprecia la evolución natural de la enfermedad hasta su total recuperación, sin que hubiese mediadn intervención médica. Correspondería a una forma de Historia Natural de este cuadro clínico. El caso N." 2 presenta una forma clínica más grave, derivada de la formación de un absceso de necesidad. La presencia de éste condicionaría la mayor parte de los síntomas generales de la enfermedad y los que frecuentemente llevan a tomar decisiones apresuradas. En esta oportunidad se esperó suficientemente y se drenó el absceso que era francamente extratorácico, con lo cual se obtuvo fácilmente el cuerpo extraño.

El Caso N. 3 se complicó por un error dizgnóstico, ya que el dato anamnéstico de la aspiración se obtuvo posteriormente al drenaje del pioneumotórax enquistado. Se aprecia así el modo en que se puede demorar la expulsión del C.E. debido a una intervención extemporánea. Se prolonga la eliminación y se corre el riesgo de que sea incompleta, requiriéndose de intervenciones posteriores de difícil realización.

E] Caso N. 4 muestra una variedad clínica interesante. El cuerpo extraño dejó de avanzar, tal vez porque tomó una vía que no le permitía salir del tórax. En este caso, la evolución natural consistió en la formación de un proceso inflamatorio crónico, que plantea una terapia en todo diferente. El diagnóstico diferencial con el secuestro pulmonar es indispensable. Descartado éste, el cuerpo extraño debe sospecharse en los procesos pulmonares crónicos con hemoptisis.

La Tabla N." 1 nos muestra el tratamiento a seguir, segín la etapa evolutiva en que se encuentre la espiga aspirada.

TABLA 1

\section{TRATAMIENTO}

$\begin{array}{ll}\text { ASPIRACION: } & \text { EXTRACCION } \\ \text { MIGRACION: } & \text { ENDOSCOPIA } \\ & \text { KINESITERAPLA } \\ \text { ABSCESO: } & \text { DRENAJE ESPONTANEO } \\ \text { NELIMOPATTA CR.: } & \text { RESECCION QUTRURGICA }\end{array}$

\section{RESUMEN}

Se presentan 4 casos de nin̄os con patología pleuropulmonar y de la pared costal de tipo inflamatorio, derivados de la aspiración de inflorescencias de gramineas (espigas de pasto) hospitalizados en el Hospital Roberto del Río.

Se analizan el diagnóstico clínico, radiológico y los procedimientos terapéuticos.

Se quiere enfatizar que lo más importante para e] diagnóstico es el dato anamnéstico y que cuando no se puede recuperar el C.E.V., por las maniobras endoscópicas o kinésicas, se recomienda esperar la evolución espontánea hacia el absceso de necesidad, el cual una vez establecido, permitirá recuperar 
íntegramente la espiga aspirada, por la pared costal.

\section{REFEREYCIAS}

'Agnilera C., Apurre f. Cuerposs extraños en la vía área. Herista Chilena de Pediatria: 43: 9, 21, 1972.

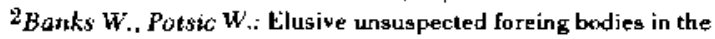
tracheptronchjal tree Clinical Pediatrica 16: 1, 31, 1977.

${ }^{3}$ Bunker P. G.: Unuecognized fureing bodies in the air and ood passages. G. P. 29: 78, 1964.

'Chopra S., Simmonds D., Cassan S., Becker S., Ben-Isaac F.: Bronchial abstruction by incorporation of aspiraled vegetable material in the bronchial wall. American Review of Respiratory Uisease 112: 717, 1975.

${ }^{5}$ Clerf $L_{\text {. }}:$ Historical aspec's of foreing bodies in the air and fond pasyagzes Southem Medical Jounial 68: 1449, 1975.

GCtery A., Ellis H., Schmidt H.: Prublems associated with aspiration of gross heads (intlorescences). J.A.M.A. 171. 11: 151, 1954.

${ }^{7}$ Cotton E., Abranu G., Vanhoutte f., Burrington J.: Remuval of aspiraled foreing bunties by inhalation and pustural drainaze. Clinical Pediatries 12: 5, 270, 1973.
Hackson C.: Foreing bodies in the Irachea, bronchi and esophagus. Laryngoscope 15: 52?, 1905.

9 Jackson C.: Enfermedades aupurativas del pulmin debidas a aspiración de cuerpo extraño, comparadas con aquellas de otra etiologia. Surgery, Gynecology and Obstetrics 42: 505, 1926.

"Ojackson C.: Grasses as foreing bodies in the Bronchus and lung The Laryngoscupe 62: 897,1952 .

11/aw D., Kosiloske A.: Managemel of Tracheubronchial foreing Indies in children: reevaluation of postural drainege and be onchusce opy. Pediatrice 58: 3, 362, 1976.

12I evnidas $f$. C.: Kadiunucleide lung geaning in the diagnosis of endubrunchial foreing bodies in children. J. Pediatr. 83: 628. 1973.

I3Moussavi H., Abbass-Zateh S.: Removal of bronchial foreing body J. Laringol. Otol. 8y: 95, 1974.

.14 Rizzardim M., Earrios R., Romero $C_{\text {.: }}$ Brisnquitis vegetal por aspiración de pepa de Sandia. Pediatria, 4: 229, 1961.

${ }^{15}$ Shinasy Majd, Mofeson H, Greensher .: Luver air wa foreing body aspiration in children. (linical Pediatrits 16: 1, 13, 1077.

${ }_{1}$ Smith $P$. S Swischuk $L$.: An clusive and of ten unsuspected cause of stridor or pneumunia. (The asophageal foreing brody, f.P. 30: $79,1965$. 